\title{
DIRECT TORQUE CONTROL OF INDUCTION MOTOR WITH EXTENDED KALMAN FILTER
}

\author{
Dinesh Pai A, L Umanand, N J Rao \\ Centre for Electronics Design and Technology \\ Indian Institute of Science, Bangalore -560012 , India \\ E - mail: adpai@cedt.iisc.ernet.in
}

\begin{abstract}
Induction motor speed control is an area of research that has been in prominence for some time now. Recent advances in this field have made it possible to replace the $D C$ motor by induction machines, even in applications that demand a fast dynamic response. Many industrial applications demand high performance speed sensorless operation, due to various reasons. Direct Torque Control (DTC) of induction motor is a popular method because of the resulting fast dynamic response of the motor, lower sensitivity to motor parameter variations and relatively low switching harmonics in the inverter. However, the present DTC approach is unsuitable for high-performance applications, especially at very low speeds, because of the need of a speed sensor for increased accuracy, system and measurement noises, and absence of any error decay mechanism. In this paper, Extended Kalman Filter $(E K F)$ is designed for stator flux and speed estimation. Estimated values along with other measured states are used for the closed loop speed sensorless control operation of the induction motor. At low speeds, where noise is predominant, performance is improved due to the filtering action of the EKF. Simulations are done and the results discussed.
\end{abstract}

Keywords: Motor control, Extended Kalman Filter, State convergence

Symbols:

$\alpha, \beta$ - stationary reference coordinates

$V_{s \alpha}, V_{s \beta}$ - stator voltage in $\alpha-\beta$ coordinates

$I_{s \alpha}, I_{s \beta}$ - stator current in $\alpha-\beta$ coordinates

$\varphi_{s \alpha}, \varphi_{s \beta}$ - stator flux in $\alpha-\beta$ coordinates

$\omega_{r}$ - motor shaft speed

$\delta$ - torque angle

$x, u, y$-system states, input, output

$T_{L}$ - load torque

$Q_{N}, R_{N}$ - process and measurement noise covariance

$P(k)$ - error covariance matrix

$K(k)$ - Kalman filter gain matrix

\section{Introduction}

Much effort has been put on the development of highperformance sensorless speed control of induction motor drives for the last few years. The main objectives of sensorless drive control are:

- Reduction of hardware complexity and cost

- Increased mechanical robustness and overall ruggedness
- Operation in hostile environments

- Higher reliability and decreased maintenance

- Increased noise immunity

- Unaffected machine inertia

In view of the above, it is desirable to eliminate sensors particularly for speed and position measurements.

A radical step in induction motor control strategies was the development of Direct Torque Control method $[1,2,3,4]$. This stator flux orientated scheme is based on the limit cycle control of both flux and torque. In principle, it uses a switching table for selecting inverter output voltage vector based on the instantaneous requirement of torque and flux, to get fast torque response and low inverter switching frequency.

For high-performance induction motor drives, information regarding the stator flux and motor speed is to be given to the controller. Conventional open loop estimation of the stator flux from the monitored voltages and currents, is not accurate enough at very low speeds owing to the considerable vóltage drop across the stator resistance, predominant effect of noises and absence of error decay mechanism [5]. This work is oriented towards the improvement to the existing Direct Torque Control method for induction motor, with an emphasis on high-performance speed sensorless operation. Extended Kalman Filter is designed for stator flux and speed estimation, which are used for the closed loop control operation of the induction motor. Known information about the noises is used to filter it out, to get good performance even at very low speeds.

\section{Principle of Direct Torque Control}

The expression for the developed torque of an induction motor is given by (1).

$$
\begin{aligned}
& T=\frac{N_{p} \cdot M}{\sigma \cdot L_{s} \cdot L_{r}} \cdot \Phi_{s} \cdot \Phi_{r} \cdot \sin \delta \\
& \text { where, } \sigma=1-\frac{M^{2}}{L_{s} L_{r}}
\end{aligned}
$$

Under normal operating conditions, the amplitude of the working flux is kept constant at the maximum value for maximum utilization of magnetic material. Hence the developed torque is proportional to the sine of the torque angle ' $\delta$ ', i.e., the angle between stator and rotor fluxes, and can be controlled by suitably changing the torque angle. Since the time constant of rotor current is large compared to stator, the stator flux is accelerated or decelerated with respect to the rotor flux to change the torque angle. 
The voltage source inverter can be modeled as shown in fig. 1, where $S_{a}, S_{b}, S_{c}$ are the switching states. Eight output voltage vectors $V_{0}$ to $V_{7}\{000,100,110,010,011$, $001,101,111\}$ as shown in fig. 2 are obtained for different switch combinations. Out of these $V_{0}$ and $V_{7}$ are zero voltage vectors. Inverter output voltage is given by the expression (2), where $\mathrm{V}_{\mathrm{dc}}$ is the $\mathrm{DC}$ - link voltage.

$$
V_{s}=\sqrt{\frac{2}{3}} \cdot V_{d c} \cdot\left(S_{a}+S_{b} \cdot e^{j 2 \pi / 3}+S_{c} \cdot e^{-j 2 \pi / 3}\right)
$$

Assuming the voltage drop across the stator resistance to be small, stator flux variation can be expressed as (3).

$$
\begin{aligned}
& \varphi_{s}=\int_{0}^{t}\left[V_{s}-I_{s} \cdot R_{s}\right] d t \\
& \Delta \varphi_{s} \cong V_{s} \cdot \Delta t
\end{aligned}
$$

This indicates that ' $\varphi_{s}$ ' moves on a locus, with constant velocity determined by the selected voltage vector and the duration ' $\Delta t$ ' for which it is applied. The output voltage vectors among $\mathrm{V}_{0}-\mathrm{V}_{7}$ are selected to change the torque angle. This is done based on the instantaneous torque requirement, ensuring the error between $\left|\varphi_{s}\right|$ and $\left|\varphi_{s}\right| *$ (reference) to be within a tolerance band. It can be seen that the selection of voltage vector also depends on the direction of $\varphi_{s}$. As shown in fig. 2 , the voltage vector changes periodically in steps of $\pi / 3$ radian. Accordingly to discriminate the direction, $\alpha-\beta$ plane is divided into six sectors $\theta(\mathbf{k})$, $k=1,2,3,4,5,6$. It can be seen from fig. 3 , that in any sector ' $k$ ', for counter clockwise rotation, voltage vectors $\mathrm{V}(\mathrm{k}+1)$ and $\mathrm{V}(\mathrm{k}+2)$ accelerates the stator flux, while $V(k-1)$ and $V(k-2)$ decelerates. Similarly $V(k), V(k+1)$ and $V(k-1)$ increases the amplitude of stator flux while $\mathrm{V}(\mathrm{k}+2), \mathrm{V}(\mathrm{k}+3)$ and $\mathrm{V}(\mathrm{k}-2)$ decreases. For clockwise rotation, reverse happens. The zero voltage vectors does not affect the stator flux substantially, with the exception of the small flux weakening due to the voltage drop across the stator resistance. Inverter output voltage vectors are selected based on the sector in which the flux lies at the instant and the instantaneous requirements of the torque and the stator flux. Two- and three-level hysterisis comparator digitizes the flux and torque errors, respectively. The digitized flux direction is determined by comparing the $\alpha-\beta$ components of the flux linkage vector with its amplitude. These digital signals, i.e., one bit of flux, two bits of torque, and three bits of sector refer the optimum switching table. Table -1 summarizes the combined action of each voltage space vector on the stator flux and the torque. As it appears from the table, for both positive and negative motor speed, an increment of torque $(\uparrow)$ is obtained by two vectors only, that are $\mathrm{V}(\mathrm{k}+1)$ and $\mathrm{V}(\mathrm{k}+2)$. Conversely, a decrement of torque $(\downarrow)$ can be obtained by applying V(k-1) or V(k-2). The radial voltage space vectors act on the torque in accordance with the motor speed direction. In this table, a single arrow means a small influence on the flux or torque variations, whilst two arrows denote a larger influence.

The hysterisis band technique leads to four possible combinations with regard to the stator flux and torque errors. Each solution affects the drive behavior in terms of torque and current ripple, switching frequency and two- or four-quadrant operation capability.
Table - 1. Effect of voltages on flux and torque

\begin{tabular}{|c|c|c|c|c|}
\hline & $T \pi, \varphi_{1} \pi$ & $T \pi, \varphi_{i} \|$ & $T \|, \varphi_{0} \pi$ & $T \rrbracket, \varphi_{t} \Downarrow$ \\
\hline Solution - A & $V(k+1)$ & $v(k+2)$ & $V_{0}, V_{7}$ & $\mathrm{~V}_{0}, \mathrm{~V}_{\mathrm{T}}$ \\
\hline Solution - B & $\mathrm{V}(\mathrm{k}+1)$ & $\mathrm{V}(\mathrm{k}+2)$ & $V(\mathbf{k})$ & $V_{0}, V_{7}$ \\
\hline Solution - C & $\mathrm{V}(\mathrm{k}+1)$ & $\mathrm{V}(\mathbf{k}+2)$ & $V(\mathbf{k})$ & $\mathrm{V}(\mathbf{k}+3)$ \\
\hline Solution - D & $\mathrm{V}(\mathrm{k}+1)$ & $\mathrm{V}(\mathrm{k}+2)$ & $\mathrm{V}(\mathrm{k}-1)$ & $V(k-2)$ \\
\hline
\end{tabular}

\begin{tabular}{|c|c|c|c|c|c|c|c|}
\hline & $\begin{array}{c}\mathrm{V} \\
(\mathbf{k}-2)\end{array}$ & $\begin{array}{c}\mathrm{V} \\
(\mathbf{k}-1)\end{array}$ & $\begin{array}{c}\mathrm{V} \\
(\mathbf{k})\end{array}$ & $\begin{array}{c}\mathrm{V} \\
(\mathbf{k}+1)\end{array}$ & $\begin{array}{c}\mathrm{V} \\
(\mathbf{k}+2)\end{array}$ & $\begin{array}{c}\mathrm{V} \\
(\mathbf{k}+3)\end{array}$ & $\begin{array}{c}\mathrm{V}_{0} \\
\text { or } \\
\mathrm{V}_{7}\end{array}$ \\
\hline$\varphi_{s}$ & $\downarrow$ & $\uparrow$ & $\uparrow \uparrow$ & $\uparrow$ & $\downarrow$ & $\downarrow \downarrow$ & $\uparrow \downarrow$ \\
\hline $\begin{array}{c}\mathrm{T} \\
\omega_{0}>0\end{array}$ & $\downarrow \downarrow$ & $\downarrow \downarrow$ & $\downarrow$ & $\uparrow$ & $\uparrow$ & $\downarrow$ & $\downarrow$ \\
\hline $\begin{array}{c}\mathrm{T} \\
\omega_{2}<0\end{array}$ & $\downarrow$ & $\downarrow$ & $\uparrow$ & $\uparrow \uparrow$ & $\uparrow \uparrow$ & $\uparrow$ & $\uparrow$ \\
\hline
\end{tabular}

Table - 2. Switching strategies

In Table -2 , four switching solutions are given. Strategies A, B and C can be used for two-quadrant operation, while strategy $D$ is suitable for four-quadrant operation.

At low speeds, it is better to select suitable active voltage vectors to reduce the torque angle rather than zero voltage vector to get fast torque response. A convenient control technique, which utilizes a different voltage space vector selection strategy, according to the operating speed range can be employed.

The schematic of speed control of induction motor using Direct Torque Control method is shown in fig. 4.

\section{Flux Estimation and Associated Problems}

Performance of the system depends greatly on the accuracy with which the stator flux and speed are estimated and these in turn depend on the accuracy of the monitored voltages and currents. Errors may occur in the monitored voltages and currents due to the following factors: phase shift in the measured values (because of sensors), magnitude errors because of conversion factors and gain, offset in the measurement system, quantisation error in the digital system, sensors and measurement noise. Conventional methods use open loop estimation of stator flux (3) by simply integrating the stator voltage $V_{s}$. For better accuracy, drop across the stator resistance is also to be considered. The main disadvantage of open loop estimation is that, it is parameter sensitive and any mismatch in the initial conditions because of the reasons mentioned above will adversely affect the system response both in transient and steady states. It may sometimes introduce output bias in steady state and cause even system instability. Any sort of error introduced in the speed estimation also can not be overcome and can lead to an increasing deviation of the result from the actual value. The reason for this is the absence of error decay mechanism. In addition, open loop speed estimation requires differentiation, which is undesirable in view of the associated noise amplification. Apart from this, the system and measurement noises predominant at low speeds may further deteriorate the system performance at low speeds. Closed-loop 
observers are better choices for improving the robustness against parameter mismatch and signal to noise ratio. Since the induction motor is nonlinear in nature, linear observers may not offer required performance over the entire operating range. Alternatively nonlinear observers can be employed. Recent developments in nonlinear control theory lead to several well-established nonlinear observer theories. Extended Kalman Filter (EKF) is one of the simplest and widely accepted nonlinear observers. It guarantees convergence to true state. EKF also enables us to use simplified models and yet get a good performance through an appropriate selection of weightages in the performance index. Its stochastic approach justifies its applicability to the control of induction motor, due to the presence of noise in high switching frequency inverter and voltage and current sensors. The working environment may also act as a source of noise. The probabilistic approach of the estimator enables us to filter out noises present in the system to the extent of whatever prior knowledge of noise characteristics we have. This filtering action of the EKF improves the system performance to a great extent at low speed operations. Consider a nonlinear stochastic process governed by (4), with process and measurement noises $w(\mathbf{k})$ and $v(\mathbf{k})$ respectively.

$$
\left.\begin{array}{l}
x(k+1)=f(x(k), u(k), w(k)) \\
y(k)=h(x(k), v(k)) \\
x \in \Re^{n} \text { and }, y \in \Re^{m}
\end{array}\right\}
$$

In practice, individual values of the noises $w(\mathrm{k})$ and $v(\mathrm{k})$ are not known at each time step. However, the state and measurement vectors can be predicted without them as in (5).

$$
\left.\begin{array}{l}
\tilde{x}(k+1)=f(\hat{x}(k), u(k), 0) \\
\tilde{y}(k)=h(\hat{x}(k), 0)
\end{array}\right\}
$$

where $\hat{x}(k)$ is some a posteriori estimate of the state. The structure of the EKF for the nonlinear system is given in (6), wherein a correction term is added to the previous predicted value to obtain the final estimate.

$$
\begin{aligned}
\hat{x}(k+1) & =f(\hat{x}(k), u(k), 0) \\
& +K(k+1)[y(k+1)-h(\tilde{x}(k+1), 0)]
\end{aligned}
$$

The filter gain ' $K$ ' is selected to minimise the performance index ' $J$ ' given in (7). A weighting matrix ' $W$ ' is included in order to assign different weightages to the state estimation errors. The observer gain will be time varying, and is to be calculated on-line.

$$
\begin{aligned}
& J=\sum_{i=1}^{n} \bar{e}_{i} \cdot W \cdot \bar{e}_{i}^{T} \\
& \text { where, } \\
& \bar{e}_{i}=E\left\{\left[x_{i}(k)-\hat{x}_{i}(k)\right]\left[x_{i}(k)-\hat{x}_{i}(k)\right]^{T}\right\} \\
& \mathrm{E}\{.\}-\text { Expectation operator }
\end{aligned}
$$

\section{Induction Motor Model and Extended Kalman Filter}

As stator flux is controlled directly, stator flux oriented model of the induction motor in $\alpha-\beta$ coordinates (stator fixed reference frame) is considered. It is assumed that the load variations are known.
Define

States, $\mathrm{x}(\mathrm{t})=\left[\begin{array}{lllll}\mathrm{x}_{1} & \mathrm{x}_{2} & \mathrm{x}_{3} & \mathrm{x}_{4} & \mathrm{x}_{5}\end{array}\right]^{\mathrm{T}}=\left[\begin{array}{lllll}I_{s \alpha} & I_{s \beta} & \varphi_{s \alpha} & \varphi_{s \beta} & \omega_{\mathrm{r}}\end{array}\right]^{\mathrm{T}}$ Input, $u(t)=\left[\begin{array}{lll}u_{1} & u_{2} & u_{3}\end{array}\right]^{T}=\left[\begin{array}{lll}V_{s \alpha} & V_{s \beta} & T_{L}\end{array}\right]^{T}$

Output, $\mathrm{y}(\mathrm{t})=\left[\begin{array}{ll}\mathrm{y}_{1} & \mathrm{y}_{2}\end{array}\right]^{\mathrm{T}}=\left[\begin{array}{ll}I_{s \alpha} & \mathrm{I}_{s \beta}\end{array}\right]^{\mathrm{T}}$

From the basic equations of induction motor, stator flux oriented continuous-time stochastic model as in (8) can be derived.

$$
\left.\begin{array}{l}
\dot{x}(t)=A(x(t)\} x(t)+B . u(t)+G . w(t) \\
y(t)=C . x(t)+v(t)
\end{array}\right\}
$$

The process noise $w(\mathrm{t})$ and measurement noise $v(\mathrm{t})$ are both continuous-time, zero mean, white, uncorrelated Gaussian noise processes and their expectation is zero. The mean and covariance of initial state and noises are known to be $x(0) \sim\left(\bar{x}_{0}, P_{0}\right), w(\mathrm{t}) \sim\left(0, Q^{c}\right)$, and, $v(\mathrm{t}) \sim$ $\left(0, R^{\circ}\right)$ respectively. Assuming a sampling time of $T_{s}$, corresponding discrete time model is given in (9).

$$
\begin{aligned}
& \left.\begin{array}{l}
x(k+1)=A_{d}\{x(k)\} x(k)+B_{d} u(k)+w(k) \\
y(k)=C_{d} x(k)+v(k)
\end{array}\right\} . \\
& \text { with, } w(k) \sim\left(0, Q_{N}\right), v(k) \sim\left(0, R_{N}\right) \\
& \text { Here, } A_{d}=e^{A . T_{s}} \cong I+A . T_{s} \\
& B_{d}=\int_{0}^{T_{d}} e^{A \tau} \cdot B \cdot d \tau \cong B \cdot T_{s} \\
& C_{d}=C \\
& w(k)=\int_{0}^{T_{d}} e^{A\left[T_{d}-\tau\right]} \cdot G \cdot w(\tau) d \tau
\end{aligned}
$$

$Q_{N}$ and $R_{N}$ are the discretised noise covariance and can be approximated as follows.

$$
\begin{aligned}
& Q_{N}=\int_{0}^{T_{d}} e^{A \tau} \cdot G \cdot Q^{c} \cdot G^{T} \cdot e^{A^{T} \tau} d \tau \equiv G \cdot Q^{c} \cdot G^{T} T_{s} \\
& R_{N}=R^{c} / T
\end{aligned}
$$

Above nonlinear model (9) is linearised around a reference trajectory. The system Jacobean is defined as in $(10)$, where $\hat{x}(k)$ is the estimated value at $k^{\text {th }}$ instant.

$$
\tilde{A}_{k+1}=\left.\frac{\partial}{\partial x}\left[A_{d}\{x(k)\} x(k)\right]\right|_{: c=x(k+1)}
$$

Recursive algorithm of the Extended Kalman Filter $[6,7]$ can be derived as below.

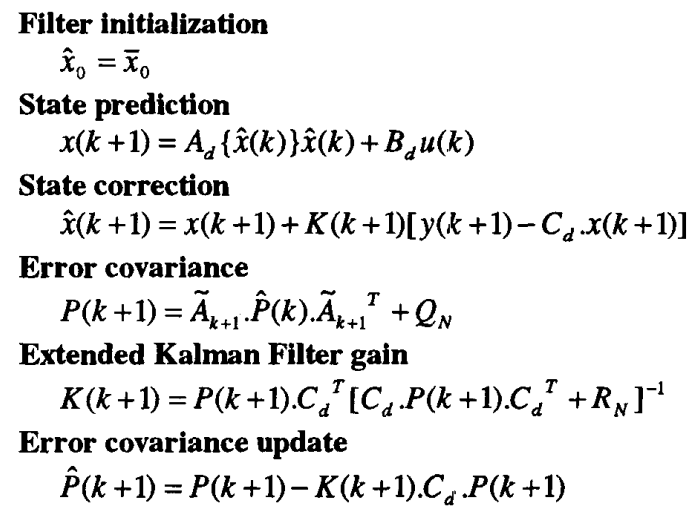

A Kalman Filter that linearizes the non-linear system about the current mean and covariance is referred to as an Extended Kalman Filter. In the first stage of calculation, states are predicted and in the second stage, 
the predicted states are corrected by making use of the measured outputs. The tuning of the EKF involves an iterative modification of covariance matrices $Q_{N}$ and $R_{N}$ in order to yield the best state estimates. The noise sources also take into account the measurement and modeling inaccuracies. The covariance matrices $Q_{N}$ and $R_{N}$ are five-by-five and two-by-two respectively. In general this would require 29 elements. Since the noises are assumed to be uncorrelated, these matrices become diagonal ones requiring only 7 elements. Further, the machine parameters in the $\alpha-\beta$ coordinates being the same, first two and second two diagonal elements of $Q_{N}$ are equal. Similarly, the two diagonal elements of $R_{N}$ are equal. Hence only 4 noise covariance elements ( 3 for $Q_{N}$ and 1 for $R_{N}$ ) need to be known in this case. An increase in $Q_{N}$ corresponds to stronger system noises, or larger uncertainty in the machine model used. This increases the error covariance $P(k+l)$ and results in higher filter gain ' $K$ '. In this case, measurements will be weighted more and the filter transient performance will be faster. On the other hand, an increase in $R_{N}$ refers to higher level of measurement noise. The filter gain ' $K$ ' will be lower in this case because of its inverse relation with $R_{N}$. Lower gain results in slower transient performance.

It should be noted that the model is discretised with only first order approximation, neglecting higher order terms. Due to this it may require very short sampling time to give a stable and accurate discrete model. A second order approximation will give better accuracy at the cost of increased computational overhead. Another issue is the inverse effect of sampling time on $R_{N}$. Lower sampling time increases $R_{N}$, adversely affecting the transient performance of the system, which is undesirable. The schematic of EKF is shown in fig. 5.

\section{Simulation Results}

Simulation studies are done for the speed control of induction motor by Direct Torque Control method with Extended Kalman Filter for the state estimation using the software tool MATLAB/SIMULINK ${ }^{\oplus}$. EKF requires any two stator line currents and the inverter DC - link voltage for processing. Keeping in view of typical high performance applications, performance specifications are set as; rise time less than $0.4 \mathrm{sec}$, steady state speed error to be less than $1 \%$ and the damping to be critical. The inverter frequency is limited to $5 \mathrm{kHz}$. A reasonable delay of $1 \mathrm{msec}$ each is considered for the current and voltage sensors. The noises are assumed to be of zero mean white Gaussian with known covariance's $Q_{N}$ and $R_{N}$. Maximum process and measurement noise levels present in the system are considered as 1 -Volt and 0.25 Ampere. Simulation for the base speed of $73 \mathrm{rad} / \mathrm{sec}$ is considered first. Actual and estimated speeds with an estimator initial state errors set to $3 \mathrm{rad} / \mathrm{sec}$ for speed and 0.1 Weber for stator flux are shown in fig. 6 and fig. 7 . The stator flux and developed torque after proper lowpass filtering are shown in fig. 8 and fig. 9. The error convergence of stator flux and speed are shown in fig. 10 and fig. 11. Secondly, we consider low speed of 1 $\mathrm{rad} / \mathrm{sec}$. Actual and estimated speeds with an estimator initial state errors set to $0.1 \mathrm{rad} / \mathrm{sec}$ for speed and 0.1
Weber for stator flux are shown in fig. 12 and fig. 13. The stator flux and developed torque after proper lowpass filtering are shown in fig. 14 and fig. 15. The error convergence of stator flux and speed are shown in fig. 16 and fig. 17. It is verified that the state error convergence is always guaranteed and the performance specifications are satisfied.

\section{Comments and Conclusions}

Sensorless Direct Torque Control of induction motor for speed control is the focus of this work. Extended Kalman Filter for stator flux and speed estimation is designed and developed for use in closed loop control. The filtering action of EKF improves the system performance, especially at low speeds. Simulation results reveal that the flux and speed tracking are good and error convergence is guaranteed. However, it is to be noted that there may be a bias problem when the assumed characteristics of the stochastic noises do not match with those of the real ones [6]. In contrast to the linear estimators, the nonlinear nature of the observed plant is likely to result in an error in the estimation. The algorithm requires computing inverse of a matrix, which increases the computation time. The results presented here are to be validated by implementing the algorithm in real time. Low cost and fast Digital Signal Processors capable of implementing relatively complex algorithms are available in the market, which makes this method suitable for high performance applications.

\section{Figures}

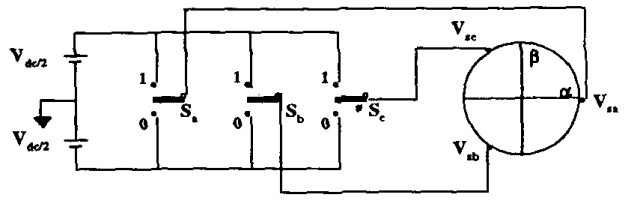

Fig. I Schematic Model of Inverter

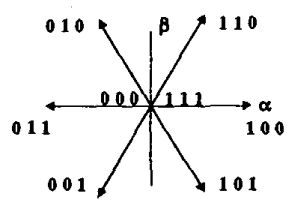

Fig. 2 Instantaneous Voltage.Vectors

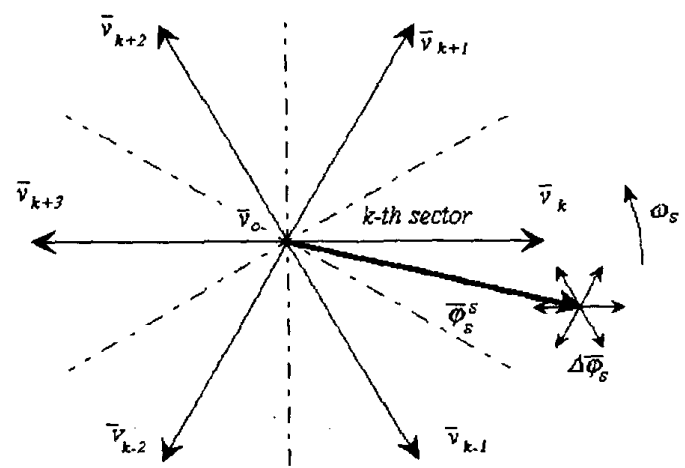

Fig. 3 Inverter Output Voltage Space Vectors and Corresponding Stator Flux Variation 


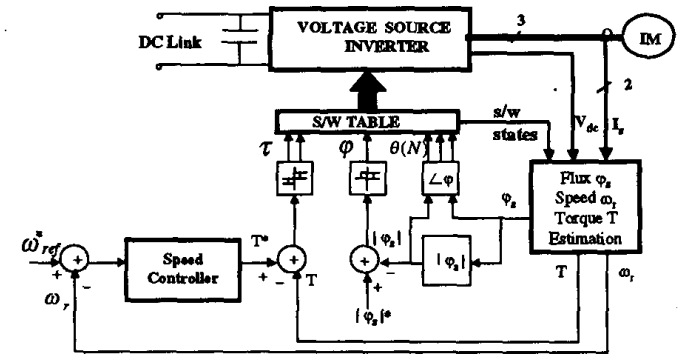

Fig. 4 Schematic of DTC with Estimator

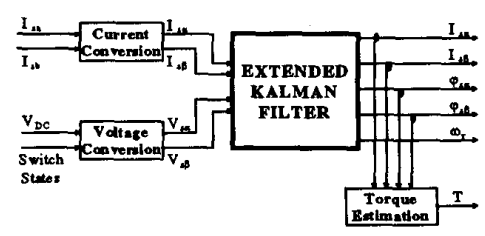

Fig. 5 Stator Flux and Speed Estimation

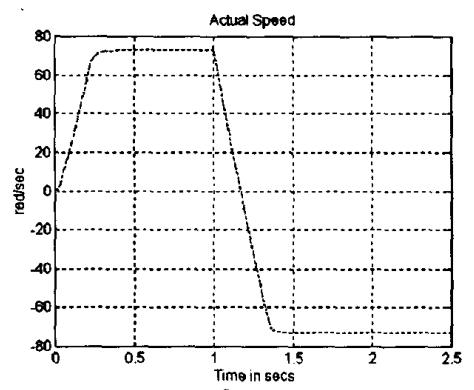

Fig. 6 Actual speed (Base speed)

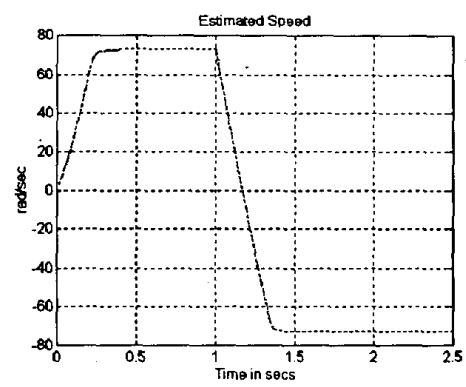

Fig. 7 Estimated speed (Base speed)

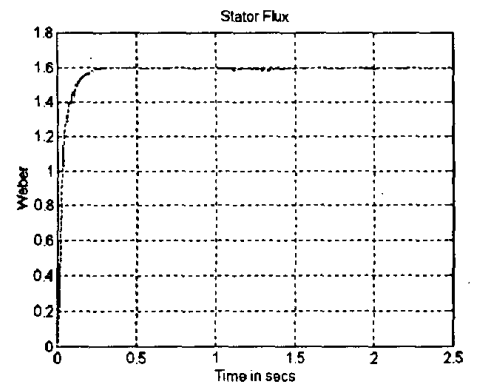

Fig. 8 Stator flux (Base speed)

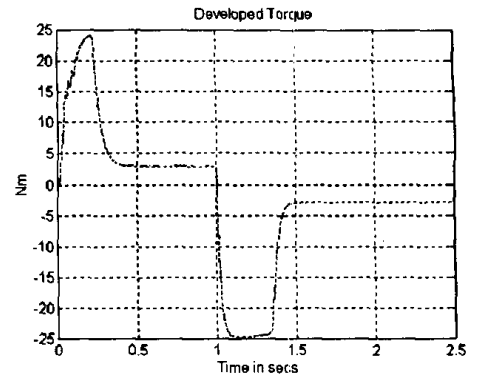

Fig. 9 Developed torque (Base speed)

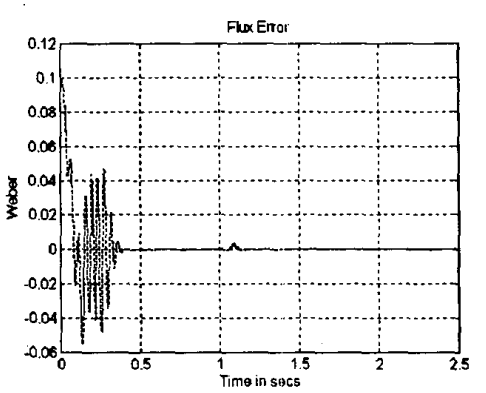

Fig. 10 Stator flux error convergence (Base speed)

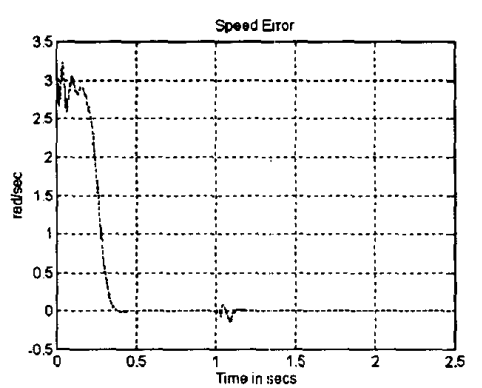

Fig. II Speed error convergence (Base speed)

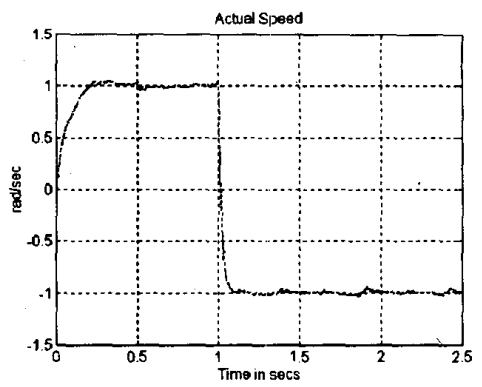

Fig. 12 Actual speed (Low speed)

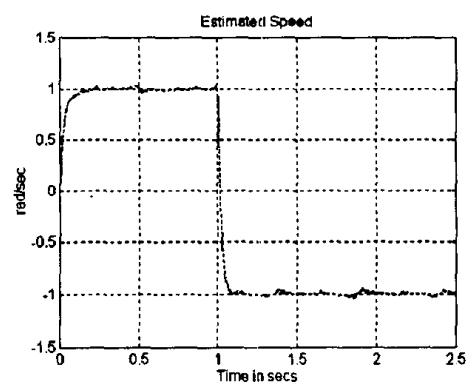

Fig. 13 Estimated speed (Low speed) 


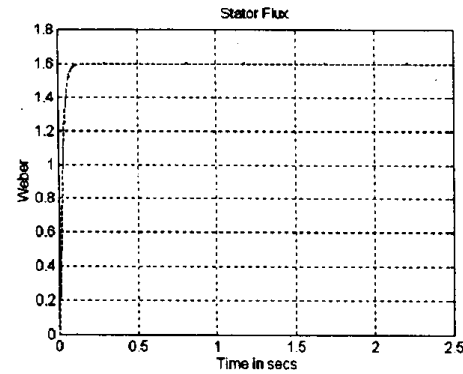

Fig. 14 Stator flux (Low speed)

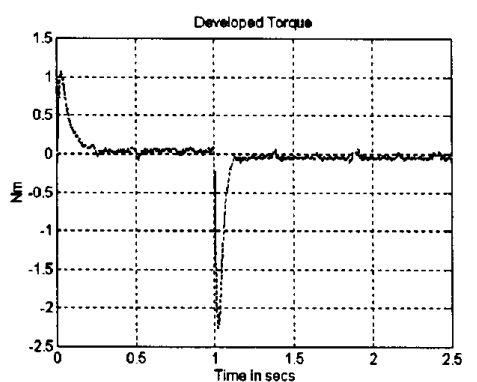

Fig. 15 Developed torque (Low speed)

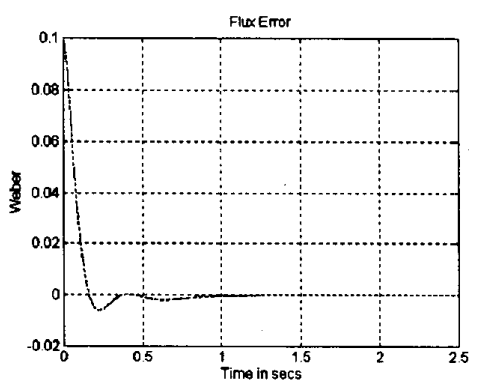

Fig. 16 Stator flux error convergence (Low speed)

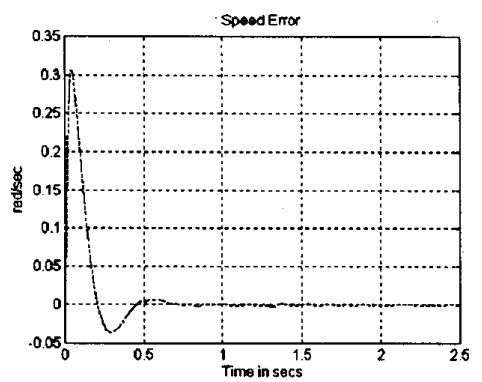

Fig. 17 Speed error convergence (Low speed)

\section{Motor parameters}

$\mathrm{L}_{s}=0.47 \mathrm{H}, \mathrm{L}_{\mathrm{r}}=0.47 \mathrm{H}, \mathrm{M}=0.44 \mathrm{H}, \mathrm{R}_{\mathrm{s}}=8 \Omega, \mathrm{R}_{\mathrm{f}}=3.6$ $\Omega, N_{p}=2,\|U\|=180 \mathrm{~V},\left\|I_{s}\right\|=4.6 \mathrm{~A}, \varphi_{s}$ [nom] $=1.6 \mathrm{~Wb}$, $\mathrm{T}_{[\text {[nom] }}=7 \mathrm{~N}-\mathrm{m}, \omega_{\mathrm{r}}$ [nom] $=73 \mathrm{rad} / \mathrm{sec}, \mathrm{J}_{\mathrm{m}}=0.06 \mathrm{Kg}-\mathrm{m}^{2}, \mathrm{~B}_{\mathrm{m}}$ $=0.04 \mathrm{~N}-\mathrm{m}-\mathrm{sec}$.

\section{References}

[1] Isao Takahashi, Toshihiko Noguchi, "A new quickresponse and high efficiency control strategy of an induction machine". IEEE Transactions on Industry Applications, vol. IA-22, no.5, September/October 1986, pp. 820-827.

[2] James N Nash, "Direct Torque Control, Induction motor vector control without an encoder". IEEE Transactions on Industry Applications, vol. 33, March/April 1997, pp. 333-341.

[3] Pekka Tiitinen, M Surendra "The next generation motor control method DTC, Direct Torque Control". Proceedings of EPE Chapter Symposium, Lausanne, Switzerland, 1994, pp. 37-43.

[4] Y A Chapuis, D Roye, J Davoine, "Principles and implementation of Direct Torque Control by stator flux orientation of an induction motor". Laboratoire d' Electro-technique de grenoble, E.N.S.I.E.G Domaine Universitaire, FRANCE.

[5] A M Walczyna, "Problems of application of Direct Flux and Torque Control methods to high power VSI-fed drives operating at low speed". Electrotechnical Institute, Department of Power Electronics, Warsaw, Poland.

[6] Young-Real Kim, Seung-Ki Sul, Min-Ho Park, "Speed sensorless vector control of induction motor using Extended Kalman Filter". IEEE Transactions on Industry Applications, vol. 30, no. 5, September /October 1994, pp. 1225 - 1233.

[7] S Beierke, P Vas, B Simor, A F Stronach, Mimare, "DSP - Controlled sensorless AC vector drives using the Extended Kalman Filter". Proceedings of PCIM'97, Nuremberg, June/1997, pp. 31 - 42.

[8] Lennart Ljung, "Asymptotic behaviour of the Extended Kalman Filter as a parameter estimator for linear systems". IEEE Transactions on Automatic Control, vol. AC - 24, no. 1, February/1979, pp.36 50. 